\title{
Reaction of Tungsten Vinylcarbene Complexes with Enamines
}

Junes Ipaktschi ${ }^{*}$, Parham Rooshenas, Ansgar Dülmer

Institute of Organic Chemistry, Justus-Liebig University, Heinrich-Buff-Ring 58, D-

35392 Giessen, Germany

Received xx. xx. 2005

\section{SUPPLEMENTARY MATERIAL}

"To whom correspondence should be addressed

FAX: $+49 / 641 / 9936309$

E-mail: junes.ipaktschi@ org.chemie.uni-giessen.de 
Figure 1 Molecular structure and atom-numbering scheme for complex 6 without $\mathrm{H}$ atoms. Thermal ellipsoids are shown at the $10 \%$ probability level

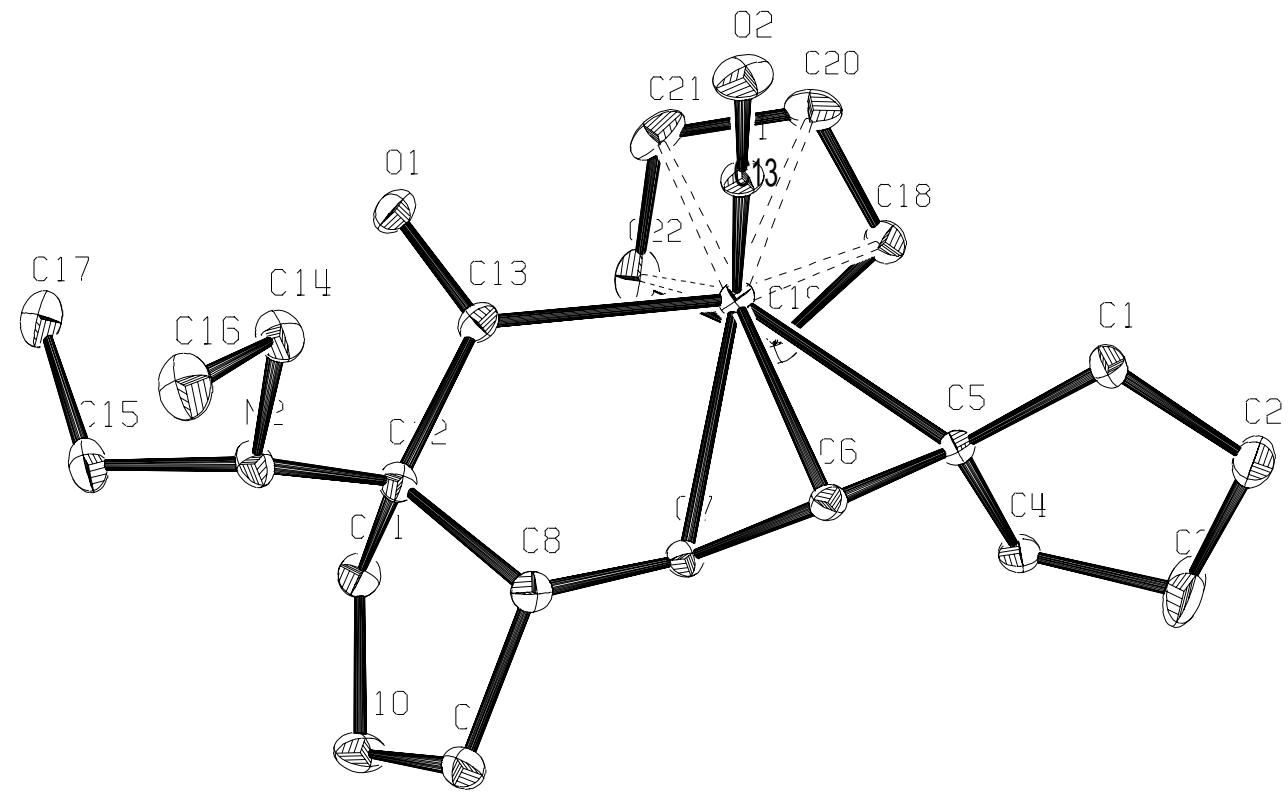


Table 1: Crystal data and structure refinement for $\mathbf{6}$

Identification code

Empirical formula

Formula weight

Temperature

Wavelength

Crystal system

Space group

Unit cell dimensions

Volume

Z

Density (calculated)

Absorption coefficient

$\mathrm{F}(000)$

Theta range for data collection

Index ranges

Reflections collected

Independent reflections

Completeness to theta $=28.05^{\circ}$

Refinement method

Data / restraints / parameters

Goodness-of-fit on $\mathrm{F}^{2}$

Final $\mathrm{R}$ indices [I $>2 \operatorname{sigma}(\mathrm{I})]$

$\mathrm{R}$ indices (all data)

Largest diff. peak and hole
6

C22 H32 N2 O2 W

540.358

293(2) K

$0.71073 \AA$

monoclinic

$\mathrm{P} 2{ }_{1} / \mathrm{c}($ No. 14$)$

$\mathrm{a}=11.330(2) \AA$

$\alpha=90^{\circ}$.

$\mathrm{b}=13.6604(10) \AA$

$\beta=92.02(10)^{\circ}$.

$\mathrm{c}=13.711(2) \AA$

$\gamma=90^{\circ}$.

2120.8(5) $\AA^{3}$

1

$1.591 \mathrm{Mg} / \mathrm{m}^{3}$

$5.462 \mathrm{~mm}^{-1}$

944

2.73 to $28.05^{\circ}$.

$-14<=\mathrm{h}<=14,-17<=\mathrm{k}<=17,-16<=\mathrm{l}<=18$

18720

$4961[\mathrm{R}(\mathrm{int})=0.0526]$

$96.6 \%$

Full-matrix least-squares on $\mathrm{F}^{2}$

4961 / 0 / 190

0.971

$\mathrm{R} 1=0.0489, \mathrm{wR} 2=0.1250$

$\mathrm{R} 1=0.0652, \mathrm{wR} 2=0.1347$

5.007 and -2.505 e. $\AA^{-3}$ 
Table 2: Atomic coordinates (x $\left.10^{4}\right)$ and equivalent isotropic displacement parameters $\left(\AA^{2} \times 10^{3}\right)$ for 6 . $\mathrm{U}(\mathrm{eq})$ is defined as one third of the trace of the orthogonalized $\mathrm{U}^{\mathrm{ij}}$ tensor.

\begin{tabular}{|c|c|c|c|c|}
\hline & $\mathrm{x}$ & $\mathrm{y}$ & $\mathrm{z}$ & $\mathrm{U}(\mathrm{eq})$ \\
\hline $\mathrm{W}(1)$ & $433(1)$ & $9088(1)$ & $2053(1)$ & $37(1)$ \\
\hline $\mathrm{N}(1)$ & $245(5)$ & $10364(5)$ & 1998(4) & $43(1)$ \\
\hline $\mathrm{N}(2)$ & $-3530(5)$ & $9280(5)$ & $2370(5)$ & $49(2)$ \\
\hline $\mathrm{O}(1)$ & $-1829(5)$ & $9195(5)$ & $866(4)$ & $68(2)$ \\
\hline $\mathrm{O}(2)$ & 129(7) & $11240(5)$ & $1879(5)$ & $71(2)$ \\
\hline $\mathrm{C}(1)$ & $-563(3)$ & $8618(3)$ & $3440(3)$ & $40(1)$ \\
\hline$C(2)$ & 313(3) & $9324(3)$ & $3701(3)$ & $41(1)$ \\
\hline$C(3)$ & $1516(3)$ & $9129(3)$ & $3496(3)$ & $43(2)$ \\
\hline$C(4)$ & 2413(4) & $9975(3)$ & $3686(3)$ & $57(2)$ \\
\hline$C(5)$ & $3384(4)$ & $9530(3)$ & $4356(3)$ & $74(3)$ \\
\hline$C(6)$ & 2991(4) & $8556(3)$ & $4609(3)$ & 133(7) \\
\hline$C(7)$ & 2117(4) & $8178(3)$ & $3860(3)$ & $54(2)$ \\
\hline $\mathrm{C}(8)$ & $-1483(4)$ & $9005(3)$ & $1682(3)$ & $46(2)$ \\
\hline $\mathrm{C}(9)$ & $-2413(6)$ & $8721(6)$ & $2446(5)$ & $42(1)$ \\
\hline $\mathrm{C}(10)$ & $-1836(2)$ & $8866(2)$ & $3483(2)$ & $45(2)$ \\
\hline $\mathrm{C}(11)$ & $-2553(2)$ & $8162(2)$ & $4128(2)$ & $60(2)$ \\
\hline$C(12)$ & $-2979(2)$ & $7304(2)$ & $3438(2)$ & $69(2)$ \\
\hline$C(13)$ & $-2592(2)$ & $7607(2)$ & $2408(2)$ & $54(2)$ \\
\hline $\mathrm{C}(14)$ & $-3352(2)$ & $10327(2)$ & 2198(2) & $63(2)$ \\
\hline$C(15)$ & $-4560(2)$ & $8863(2)$ & $1838(2)$ & $66(2)$ \\
\hline$C(16)$ & $-4263(2)$ & $10949(2)$ & $2723(2)$ & $78(3)$ \\
\hline$C(17)$ & $-4511(2)$ & $8778(2)$ & $708(2)$ & $85(3)$ \\
\hline $\mathrm{C}(18)$ & $2156(2)$ & $8316(2)$ & $1501(2)$ & $58(2)$ \\
\hline C(19) & $1323(2)$ & $7563(2)$ & $1595(2)$ & $56(2)$ \\
\hline$C(20)$ & $1738(2)$ & $8962(2)$ & $780(2)$ & $76(3)$ \\
\hline $\mathrm{C}(21)$ & $632(2)$ & $8606(2)$ & $439(2)$ & $80(4)$ \\
\hline $\mathrm{C}(22)$ & $369(2)$ & $7767(2)$ & $938(2)$ & $73(3)$ \\
\hline
\end{tabular}


Table 3: Bond lengths $[\AA]$ and angles $\left[^{\circ}\right]$ for 6 .

\begin{tabular}{|c|c|}
\hline $\mathrm{W}(1)-\mathrm{N}(1)$ & $1.758(6)$ \\
\hline $\mathrm{W}(1)-\mathrm{C}(8)$ & $2.215(4)$ \\
\hline $\mathrm{W}(1)-\mathrm{C}(3)$ & $2.293(4)$ \\
\hline $\mathrm{W}(1)-\mathrm{C}(2)$ & $2.292(4)$ \\
\hline $\mathrm{W}(1)-\mathrm{C}(21)$ & $2.326(3)$ \\
\hline $\mathrm{W}(1)-\mathrm{C}(20)$ & $2.332(3)$ \\
\hline $\mathrm{W}(1)-\mathrm{C}(1)$ & $2.337(4)$ \\
\hline $\mathrm{W}(1)-\mathrm{C}(22)$ & $2.365(3)$ \\
\hline $\mathrm{W}(1)-\mathrm{C}(18)$ & $2.367(3)$ \\
\hline $\mathrm{W}(1)-\mathrm{C}(19)$ & $2.407(3)$ \\
\hline $\mathrm{N}(1)-\mathrm{O}(2)$ & $1.214(10)$ \\
\hline $\mathrm{N}(2)-\mathrm{C}(14)$ & $1.465(7)$ \\
\hline $\mathrm{N}(2)-\mathrm{C}(15)$ & $1.470(7)$ \\
\hline $\mathrm{N}(2)-\mathrm{C}(9)$ & $1.478(9)$ \\
\hline $\mathrm{O}(1)-\mathrm{C}(8)$ & $1.200(7)$ \\
\hline $\mathrm{C}(1)-\mathrm{C}(2)$ & 1.4210 \\
\hline $\mathrm{C}(1)-\mathrm{C}(10)$ & $1.484(5)$ \\
\hline$C(2)-C(3)$ & 1.4270 \\
\hline$C(3)-C(7)$ & $1.541(6)$ \\
\hline$C(3)-C(4)$ & $1.555(6)$ \\
\hline$C(4)-C(5)$ & 1.5341 \\
\hline$C(5)-C(6)$ & 1.4498 \\
\hline$C(6)-C(7)$ & 1.4932 \\
\hline$C(8)-C(9)$ & $1.562(8)$ \\
\hline $\mathrm{C}(9)-\mathrm{C}(13)$ & $1.536(8)$ \\
\hline $\mathrm{C}(9)-\mathrm{C}(10)$ & $1.556(7)$ \\
\hline $\mathrm{C}(10)-\mathrm{C}(11)$ & 1.5551 \\
\hline$C(11)-C(12)$ & 1.5708 \\
\hline $\mathrm{C}(12)-\mathrm{C}(13)$ & 1.5495 \\
\hline$C(14)-C(16)$ & 1.5360 \\
\hline$C(15)-C(17)$ & 1.5562 \\
\hline $\mathrm{C}(18)-\mathrm{C}(20)$ & 1.3960 \\
\hline $\mathrm{C}(18)-\mathrm{C}(19)$ & 1.4049 \\
\hline$C(19)-C(22)$ & 1.4112 \\
\hline $\mathrm{C}(20)-\mathrm{C}(21)$ & 1.4081 \\
\hline$C(21)-C(22)$ & 1.3732 \\
\hline
\end{tabular}




\begin{tabular}{|c|c|}
\hline $\mathrm{N}(1)-\mathrm{W}(1)-\mathrm{C}(8)$ & $85.7(2)$ \\
\hline $\mathrm{N}(1)-\mathrm{W}(1)-\mathrm{C}(3)$ & $94.2(2)$ \\
\hline $\mathrm{C}(8)-\mathrm{W}(1)-\mathrm{C}(3)$ & $133.62(17)$ \\
\hline $\mathrm{N}(1)-\mathrm{W}(1)-\mathrm{C}(2)$ & $83.7(2)$ \\
\hline $\mathrm{C}(8)-\mathrm{W}(1)-\mathrm{C}(2)$ & $98.14(18)$ \\
\hline $\mathrm{C}(3)-\mathrm{W}(1)-\mathrm{C}(2)$ & $36.27(6)$ \\
\hline $\mathrm{N}(1)-\mathrm{W}(1)-\mathrm{C}(21)$ & $104.8(2)$ \\
\hline $\mathrm{C}(8)-\mathrm{W}(1)-\mathrm{C}(21)$ & $83.93(16)$ \\
\hline $\mathrm{C}(3)-\mathrm{W}(1)-\mathrm{C}(21)$ & $139.56(12)$ \\
\hline $\mathrm{C}(2)-\mathrm{W}(1)-\mathrm{C}(21)$ & $171.36(13)$ \\
\hline $\mathrm{N}(1)-\mathrm{W}(1)-\mathrm{C}(20)$ & $96.9(2)$ \\
\hline C(8)-W(1)-C(20) & $117.80(14)$ \\
\hline $\mathrm{C}(3)-\mathrm{W}(1)-\mathrm{C}(20)$ & $108.28(12)$ \\
\hline $\mathrm{C}(2)-\mathrm{W}(1)-\mathrm{C}(20)$ & $144.03(12)$ \\
\hline $\mathrm{C}(21)-\mathrm{W}(1)-\mathrm{C}(20)$ & 35.2 \\
\hline $\mathrm{N}(1)-\mathrm{W}(1)-\mathrm{C}(1)$ & $104.2(2)$ \\
\hline $\mathrm{C}(8)-\mathrm{W}(1)-\mathrm{C}(1)$ & $71.05(16)$ \\
\hline $\mathrm{C}(3)-\mathrm{W}(1)-\mathrm{C}(1)$ & $64.07(10)$ \\
\hline $\mathrm{C}(2)-\mathrm{W}(1)-\mathrm{C}(1)$ & $35.74(6)$ \\
\hline $\mathrm{C}(21)-\mathrm{W}(1)-\mathrm{C}(1)$ & $139.68(13)$ \\
\hline $\mathrm{C}(20)-\mathrm{W}(1)-\mathrm{C}(1)$ & $157.82(13)$ \\
\hline $\mathrm{N}(1)-\mathrm{W}(1)-\mathrm{C}(22)$ & 136.79(19) \\
\hline $\mathrm{C}(8)-\mathrm{W}(1)-\mathrm{C}(22)$ & $78.76(15)$ \\
\hline $\mathrm{C}(3)-\mathrm{W}(1)-\mathrm{C}(22)$ & $125.46(13)$ \\
\hline $\mathrm{C}(2)-\mathrm{W}(1)-\mathrm{C}(22)$ & $138.03(13)$ \\
\hline $\mathrm{C}(21)-\mathrm{W}(1)-\mathrm{C}(22)$ & 34.0 \\
\hline $\mathrm{C}(20)-\mathrm{W}(1)-\mathrm{C}(22)$ & $57.73(6)$ \\
\hline $\mathrm{C}(1)-\mathrm{W}(1)-\mathrm{C}(22)$ & $108.18(13)$ \\
\hline $\mathrm{N}(1)-\mathrm{W}(1)-\mathrm{C}(18)$ & $121.9(2)$ \\
\hline $\mathrm{C}(8)-\mathrm{W}(1)-\mathrm{C}(18)$ & $135.80(14)$ \\
\hline $\mathrm{C}(3)-\mathrm{W}(1)-\mathrm{C}(18)$ & $82.19(13)$ \\
\hline $\mathrm{C}(2)-\mathrm{W}(1)-\mathrm{C}(18)$ & $117.15(14)$ \\
\hline $\mathrm{C}(21)-\mathrm{W}(1)-\mathrm{C}(18)$ & $57.42(9)$ \\
\hline $\mathrm{C}(20)-\mathrm{W}(1)-\mathrm{C}(18)$ & 34.6 \\
\hline $\mathrm{C}(1)-\mathrm{W}(1)-\mathrm{C}(18)$ & $124.42(14)$ \\
\hline $\mathrm{C}(22)-\mathrm{W}(1)-\mathrm{C}(18)$ & $57.33(7)$ \\
\hline $\mathrm{N}(1)-\mathrm{W}(1)-\mathrm{C}(19)$ & $154.0(2)$ \\
\hline $\mathrm{C}(8)-\mathrm{W}(1)-\mathrm{C}(19)$ & $108.20(13)$ \\
\hline $\mathrm{C}(3)-\mathrm{W}(1)-\mathrm{C}(19)$ & $91.69(13)$ \\
\hline
\end{tabular}




\begin{tabular}{|c|c|}
\hline $\mathrm{C}(2)-\mathrm{W}(1)-\mathrm{C}(19)$ & $114.76(13)$ \\
\hline C(21)-W(1)-C(19) & $56.78(7)$ \\
\hline C(20)-W(1)-C(19) & $57.25(6)$ \\
\hline $\mathrm{C}(1)-\mathrm{W}(1)-\mathrm{C}(19)$ & $101.13(13)$ \\
\hline C(22)-W(1)-C(19) & 34.4 \\
\hline C(18)-W(1)-C(19) & 34.2 \\
\hline $\mathrm{O}(2)-\mathrm{N}(1)-\mathrm{W}(1)$ & $174.6(5)$ \\
\hline $\mathrm{C}(14)-\mathrm{N}(2)-\mathrm{C}(15)$ & $114.2(5)$ \\
\hline $\mathrm{C}(14)-\mathrm{N}(2)-\mathrm{C}(9)$ & $113.2(5)$ \\
\hline $\mathrm{C}(15)-\mathrm{N}(2)-\mathrm{C}(9)$ & $119.9(5)$ \\
\hline $\mathrm{C}(2)-\mathrm{C}(1)-\mathrm{C}(10)$ & $120.39(19)$ \\
\hline $\mathrm{C}(2)-\mathrm{C}(1)-\mathrm{W}(1)$ & $70.41(11)$ \\
\hline $\mathrm{C}(10)-\mathrm{C}(1)-\mathrm{W}(1)$ & $117.8(2)$ \\
\hline $\mathrm{C}(1)-\mathrm{C}(2)-\mathrm{C}(3)$ & 119.2 \\
\hline $\mathrm{C}(1)-\mathrm{C}(2)-\mathrm{W}(1)$ & $73.85(12)$ \\
\hline $\mathrm{C}(3)-\mathrm{C}(2)-\mathrm{W}(1)$ & $71.89(14)$ \\
\hline $\mathrm{C}(2)-\mathrm{C}(3)-\mathrm{C}(7)$ & $120.6(2)$ \\
\hline $\mathrm{C}(2)-\mathrm{C}(3)-\mathrm{C}(4)$ & $116.8(2)$ \\
\hline $\mathrm{C}(7)-\mathrm{C}(3)-\mathrm{C}(4)$ & 107.1(3) \\
\hline $\mathrm{C}(2)-\mathrm{C}(3)-\mathrm{W}(1)$ & $71.84(12)$ \\
\hline $\mathrm{C}(7)-\mathrm{C}(3)-\mathrm{W}(1)$ & $118.4(3)$ \\
\hline $\mathrm{C}(4)-\mathrm{C}(3)-\mathrm{W}(1)$ & 119.3(3) \\
\hline$C(5)-C(4)-C(3)$ & $104.8(2)$ \\
\hline$C(6)-C(5)-C(4)$ & 106.7 \\
\hline$C(5)-C(6)-C(7)$ & 110.8 \\
\hline$C(6)-C(7)-C(3)$ & $101.9(2)$ \\
\hline $\mathrm{O}(1)-\mathrm{C}(8)-\mathrm{C}(9)$ & $118.2(5)$ \\
\hline $\mathrm{O}(1)-\mathrm{C}(8)-\mathrm{W}(1)$ & 119.2(4) \\
\hline $\mathrm{C}(9)-\mathrm{C}(8)-\mathrm{W}(1)$ & $122.5(3)$ \\
\hline $\mathrm{N}(2)-\mathrm{C}(9)-\mathrm{C}(13)$ & $113.4(5)$ \\
\hline $\mathrm{N}(2)-\mathrm{C}(9)-\mathrm{C}(10)$ & $109.3(5)$ \\
\hline$C(13)-C(9)-C(10)$ & $102.0(5)$ \\
\hline $\mathrm{N}(2)-\mathrm{C}(9)-\mathrm{C}(8)$ & $114.9(6)$ \\
\hline $\mathrm{C}(13)-\mathrm{C}(9)-\mathrm{C}(8)$ & $108.4(5)$ \\
\hline $\mathrm{C}(10)-\mathrm{C}(9)-\mathrm{C}(8)$ & $108.1(4)$ \\
\hline$C(1)-C(10)-C(9)$ & $108.2(3)$ \\
\hline$C(1)-C(10)-C(11)$ & $114.1(2)$ \\
\hline C(9)-C(10)-C(11) & $103.3(3)$ \\
\hline$C(10)-C(11)-C(12)$ & 105.9 \\
\hline
\end{tabular}




$\begin{array}{lc}\mathrm{C}(13)-\mathrm{C}(12)-\mathrm{C}(11) & 105.0 \\ \mathrm{C}(9)-\mathrm{C}(13)-\mathrm{C}(12) & 106.0(3) \\ \mathrm{N}(2)-\mathrm{C}(14)-\mathrm{C}(16) & 111.5(3) \\ \mathrm{N}(2)-\mathrm{C}(15)-\mathrm{C}(17) & 117.8(3) \\ \mathrm{C}(20)-\mathrm{C}(18)-\mathrm{C}(19) & 108.4 \\ \mathrm{C}(20)-\mathrm{C}(18)-\mathrm{W}(1) & 71.38(8) \\ \mathrm{C}(19)-\mathrm{C}(18)-\mathrm{W}(1) & 74.47(7) \\ \mathrm{C}(18)-\mathrm{C}(19)-\mathrm{C}(22) & 107.4 \\ \mathrm{C}(18)-\mathrm{C}(19)-\mathrm{W}(1) & 71.31(7) \\ \mathrm{C}(22)-\mathrm{C}(19)-\mathrm{W}(1) & 71.16(6) \\ \mathrm{C}(18)-\mathrm{C}(20)-\mathrm{C}(21) & 107.0 \\ \mathrm{C}(18)-\mathrm{C}(20)-\mathrm{W}(1) & 74.06(7) \\ \mathrm{C}(21)-\mathrm{C}(20)-\mathrm{W}(1) & 72.17(8) \\ \mathrm{C}(22)-\mathrm{C}(21)-\mathrm{C}(20) & 109.2 \\ \mathrm{C}(22)-\mathrm{C}(21)-\mathrm{W}(1) & 74.52(8) \\ \mathrm{C}(20)-\mathrm{C}(21)-\mathrm{W}(1) & 72.64(10) \\ \mathrm{C}(21)-\mathrm{C}(22)-\mathrm{C}(19) & 107.9 \\ \mathrm{C}(21)-\mathrm{C}(22)-\mathrm{W}(1) & 71.44(7) \\ \mathrm{C}(19)-\mathrm{C}(22)-\mathrm{W}(1) & 74.45(7) \\ & \end{array}$

Symmetry transformations used to generate equivalent atoms: 
Table 4: Anisotropic displacement parameters $\left(\AA^{2} \mathrm{x} 10^{3}\right)$ for 6.

The anisotropic displacement factor exponent takes the form: $-2 \pi^{2}\left[h^{2} a^{* 2} U^{11}+\ldots+2 h k a^{*} b^{*} U^{12}\right]$

\begin{tabular}{|c|c|c|c|c|c|c|}
\hline & $\mathrm{U}^{11}$ & $\mathrm{U}^{22}$ & $\mathrm{U}^{33}$ & $\mathrm{U}^{23}$ & $\mathrm{U}^{13}$ & $\mathrm{U}^{12}$ \\
\hline $\mathrm{W}(1)$ & $40(1)$ & $36(1)$ & $33(1)$ & $-1(1)$ & $2(1)$ & $6(1)$ \\
\hline $\mathrm{N}(1)$ & $56(3)$ & $28(3)$ & $44(3)$ & $3(2)$ & $-1(2)$ & $4(2)$ \\
\hline $\mathrm{N}(2)$ & $40(3)$ & $37(4)$ & $71(4)$ & $-2(3)$ & $3(3)$ & $-2(2)$ \\
\hline $\mathrm{O}(1)$ & $57(3)$ & $98(6)$ & $48(3)$ & $14(3)$ & $-7(2)$ & $9(3)$ \\
\hline $\mathrm{O}(2)$ & 113(5) & $31(4)$ & $68(4)$ & $4(3)$ & $-6(3)$ & $-4(3)$ \\
\hline $\mathrm{C}(1)$ & $49(3)$ & $31(4)$ & $42(3)$ & $-3(3)$ & $0(3)$ & $-8(3)$ \\
\hline$C(2)$ & $48(3)$ & $43(4)$ & $30(3)$ & $-4(2)$ & $0(2)$ & $-5(3)$ \\
\hline$C(3)$ & $41(3)$ & $45(4)$ & $41(3)$ & $8(3)$ & $-3(2)$ & $-2(3)$ \\
\hline$C(4)$ & $48(4)$ & $57(6)$ & $65(5)$ & $8(4)$ & $-7(3)$ & $-15(3)$ \\
\hline$C(5)$ & $71(5)$ & $76(7)$ & $73(6)$ & $3(5)$ & $-22(5)$ & $-6(5)$ \\
\hline$C(6)$ & $128(11)$ & $98(11)$ & $166(14)$ & $41(10)$ & $-102(11)$ & $-23(9)$ \\
\hline$C(7)$ & $56(4)$ & $45(5)$ & $60(4)$ & $12(3)$ & $-2(3)$ & $-1(3)$ \\
\hline $\mathrm{C}(8)$ & $52(4)$ & $44(5)$ & $42(3)$ & $-2(3)$ & $-2(3)$ & $10(3)$ \\
\hline $\mathrm{C}(9)$ & $44(3)$ & $32(4)$ & $49(4)$ & $-3(3)$ & $-2(3)$ & $-2(3)$ \\
\hline$C(10)$ & $51(4)$ & $40(4)$ & $43(3)$ & $-6(3)$ & $2(3)$ & $-1(3)$ \\
\hline $\mathrm{C}(11)$ & $54(4)$ & $77(7)$ & $51(4)$ & $0(4)$ & $8(3)$ & $-11(4)$ \\
\hline $\mathrm{C}(12)$ & $88(6)$ & $51(6)$ & $66(5)$ & $9(4)$ & $9(4)$ & $-19(5)$ \\
\hline$C(13)$ & $62(4)$ & $36(5)$ & $63(5)$ & $-6(3)$ & $-4(3)$ & $-3(3)$ \\
\hline$C(14)$ & $59(5)$ & $43(5)$ & $89(6)$ & $2(4)$ & $3(4)$ & $0(4)$ \\
\hline$C(15)$ & $43(4)$ & $60(6)$ & $95(7)$ & $-2(5)$ & $-5(4)$ & $-5(3)$ \\
\hline$C(16)$ & $64(5)$ & $51(7)$ & 119(9) & $-6(5)$ & $-2(6)$ & $16(4)$ \\
\hline$C(17)$ & $63(6)$ & $106(10)$ & $85(7)$ & $3(6)$ & $-21(5)$ & $-2(6)$ \\
\hline$C(18)$ & $46(4)$ & $68(6)$ & $60(4)$ & $-5(4)$ & $15(3)$ & $9(4)$ \\
\hline$C(19)$ & $67(5)$ & $51(6)$ & $52(4)$ & $-4(3)$ & $12(3)$ & $17(4)$ \\
\hline$C(20)$ & $87(7)$ & $80(8)$ & $64(6)$ & $5(5)$ & $37(5)$ & $13(5)$ \\
\hline $\mathrm{C}(21)$ & $98(7)$ & 108(9) & $35(4)$ & $-10(5)$ & $4(4)$ & $52(7)$ \\
\hline $\mathrm{C}(22)$ & $71(5)$ & $87(8)$ & $60(5)$ & $-43(5)$ & 1(4) & $16(5)$ \\
\hline
\end{tabular}


Figure 2 Molecular structure and atom-numbering scheme for complex 11 without $\mathrm{H}$ atoms. Thermal ellipsoids are shown at the $10 \%$ probability level

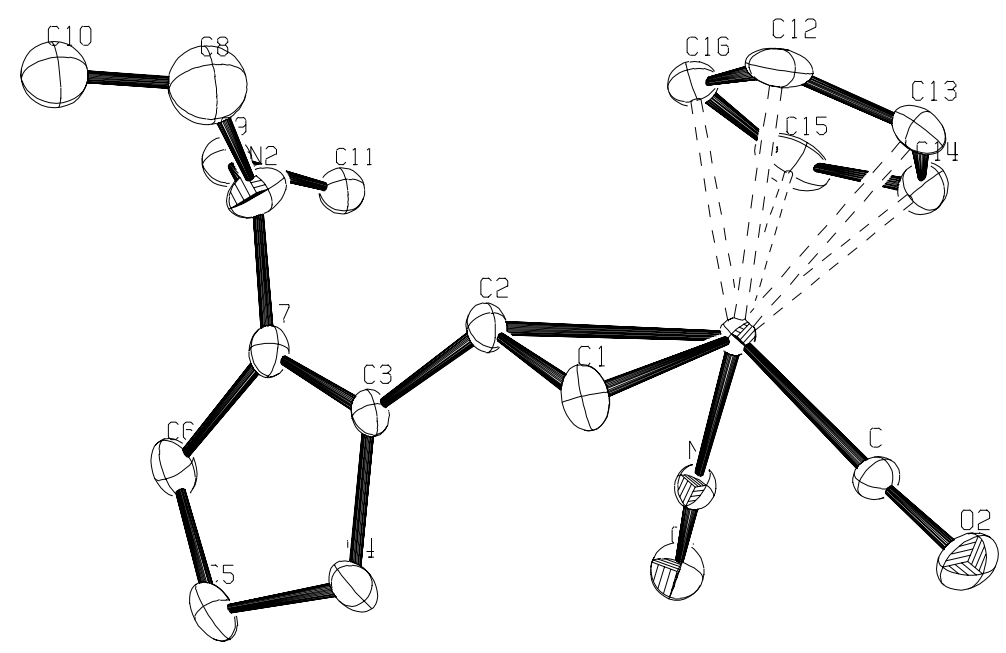


Table 1: Crystal data and structure refinement for $\mathbf{1 1}$

Identification code

Empirical formula

Formula weight

Temperature

Wavelength

Crystal system

Space group

Unit cell dimensions

Volume

$\mathrm{Z}$

Density (calculated)

Absorption coefficient

$\mathrm{F}(000)$

Theta range for data collection

Index ranges

Reflections collected

Independent reflections

Completeness to theta $=28.04^{\circ}$

Refinement method

Data / restraints / parameters

Goodness-of-fit on $\mathrm{F}^{2}$

Final $\mathrm{R}$ indices $[\mathrm{I}>2 \operatorname{sigma}(\mathrm{I})]$

$\mathrm{R}$ indices (all data)

Largest diff. peak and hole
11

C17 H24 N2 O2 W

472.24

293(2) K

$0.71073 \AA$

monoclinic

$\mathrm{P} 2{ }_{1} / \mathrm{c}$ (No.14)

$\mathrm{a}=16.0910(18) \AA \quad \alpha=90^{\circ}$.

$\mathrm{b}=6.7543(5) \AA \quad \beta=104.422(13)^{\circ}$.

$\mathrm{c}=16.8063(18) \AA \quad \gamma=90^{\circ}$.

$1769.0(3) \AA^{3}$

1

$1.769 \mathrm{Mg} / \mathrm{m}^{3}$

$6.538 \mathrm{~mm}^{-1}$

916

3.40 to $28.04^{\circ}$

$-21<=\mathrm{h}<=21,-8<=\mathrm{k}<=8,-22<=\mathrm{l}<=22$

15178

$4007[\mathrm{R}(\mathrm{int})=0.0286]$

$93.7 \%$

Full-matrix least-squares on $\mathrm{F}^{2}$

4007 / 0 / 173

1.146

$\mathrm{R} 1=0.0312, \mathrm{wR} 2=0.0802$

$\mathrm{R} 1=0.0477, \mathrm{wR} 2=0.0831$

1.259 and -0.522 e. $\AA^{-3}$ 
Table 2: Atomic coordinates $\left(\mathrm{x} 10^{4}\right)$ and equivalent isotropic displacement parameters $\left(\AA^{2} \times 10^{3}\right)$ for $\mathbf{1 1}$. U(eq) is defined as one third of the trace of the orthogonalized $U^{i j}$ tensor.

\begin{tabular}{|c|c|c|c|c|}
\hline & $\mathrm{x}$ & $\mathrm{y}$ & $\mathrm{z}$ & $\mathrm{U}(\mathrm{eq})$ \\
\hline W & $1443(1)$ & $3703(1)$ & $4524(1)$ & $49(1)$ \\
\hline $\mathrm{N}$ & $1608(3)$ & $6147(10)$ & 4937(3) & $62(1)$ \\
\hline $\mathrm{O}(1)$ & $1628(5)$ & $7855(11)$ & $5153(5)$ & $102(2)$ \\
\hline $\mathrm{C}$ & $1409(5)$ & $2624(12)$ & $5605(4)$ & $64(2)$ \\
\hline $\mathrm{O}(2)$ & $1356(5)$ & $1988(12)$ & $6210(4)$ & $107(2)$ \\
\hline $\mathrm{C}(11)$ & $1004(8)$ & $3080(30)$ & $3118(5)$ & $118(5)$ \\
\hline$C(12)$ & $925(8)$ & $1360(20)$ & $3478(7)$ & $120(4)$ \\
\hline$C(13)$ & $354(7)$ & $1450(20)$ & $3910(7)$ & $110(4)$ \\
\hline$C(14)$ & $21(5)$ & $3270(30)$ & $3867(6)$ & 113(5) \\
\hline$C(15)$ & 371(9) & $4400(20)$ & $3371(8)$ & $136(5)$ \\
\hline $\mathrm{C}(1)$ & $2734(5)$ & $2224(14)$ & $5027(6)$ & $79(2)$ \\
\hline$C(2)$ & 2804(4) & $3371(11)$ & $4358(5)$ & $63(2)$ \\
\hline $\mathrm{C}(21)$ & $3321(4)$ & $5178(11)$ & $4418(4)$ & $59(2)$ \\
\hline$C(22)$ & $3531(4)$ & $6090(13)$ & $3809(5)$ & $70(2)$ \\
\hline$C(23)$ & $4106(6)$ & $7856(17)$ & $4102(7)$ & $96(3)$ \\
\hline $\mathrm{C}(24)$ & $4299(5)$ & $7755(17)$ & 4995(7) & $97(3)$ \\
\hline$C(25)$ & $3735(5)$ & 6131(16) & $5222(5)$ & $88(2)$ \\
\hline $\mathrm{N}(2)$ & $3235(6)$ & $5517(13)$ & $2954(5)$ & $102(3)$ \\
\hline $\mathrm{C}(31)$ & $3046(13)$ & $7050(30)$ & 2388(13) & $94(5)$ \\
\hline $\mathrm{C}(32)$ & $2298(11)$ & $8060(30)$ & $2434(11)$ & $84(4)$ \\
\hline$C(41)$ & 3507 & 3631 & 2706 & $195(15)$ \\
\hline$C(42)$ & 4257 & 3685 & 2543 & 136(9) \\
\hline
\end{tabular}


Table 3: Bond lengths $[\AA]$ and angles $\left[{ }^{\circ}\right]$ for $\mathbf{1 1}$.

\begin{tabular}{|c|c|}
\hline $\mathrm{W}-\mathrm{N}$ & $1.786(7)$ \\
\hline $\mathrm{W}-\mathrm{C}$ & $1.972(6)$ \\
\hline W-C(1) & $2.270(7)$ \\
\hline W-C(2) & $2.286(6)$ \\
\hline W-C(14) & $2.298(8)$ \\
\hline $\mathrm{W}-\mathrm{C}(15)$ & $2.300(8)$ \\
\hline W-C(11) & $2.329(8)$ \\
\hline W-C(13) & $2.355(9)$ \\
\hline W-C(12) & $2.355(9)$ \\
\hline $\mathrm{N}-\mathrm{O}(1)$ & $1.207(8)$ \\
\hline $\mathrm{C}-\mathrm{O}(2)$ & $1.126(8)$ \\
\hline $\mathrm{C}(11)-\mathrm{C}(12)$ & $1.327(18)$ \\
\hline $\mathrm{C}(11)-\mathrm{C}(15)$ & $1.49(2)$ \\
\hline $\mathrm{C}(12)-\mathrm{C}(13)$ & $1.305(16)$ \\
\hline $\mathrm{C}(13)-\mathrm{C}(14)$ & $1.334(18)$ \\
\hline $\mathrm{C}(14)-\mathrm{C}(15)$ & $1.355(19)$ \\
\hline $\mathrm{C}(1)-\mathrm{C}(2)$ & $1.393(11)$ \\
\hline $\mathrm{C}(2)-\mathrm{C}(21)$ & $1.467(10)$ \\
\hline $\mathrm{C}(21)-\mathrm{C}(22)$ & $1.309(10)$ \\
\hline $\mathrm{C}(21)-\mathrm{C}(25)$ & $1.495(11)$ \\
\hline $\mathrm{C}(22)-\mathrm{N}(2)$ & $1.449(11)$ \\
\hline $\mathrm{C}(22)-\mathrm{C}(23)$ & $1.515(12)$ \\
\hline $\mathrm{C}(23)-\mathrm{C}(24)$ & $1.455(14)$ \\
\hline $\mathrm{C}(24)-\mathrm{C}(25)$ & $1.532(12)$ \\
\hline $\mathrm{N}(2)-\mathrm{C}(31)$ & $1.39(2)$ \\
\hline $\mathrm{N}(2)-\mathrm{C}(41)$ & $1.442(9)$ \\
\hline $\mathrm{C}(31)-\mathrm{C}(32)$ & $1.40(3)$ \\
\hline$C(41)-C(42)$ & $1.30270(14)$ \\
\hline N-W-C & 91.1(3) \\
\hline $\mathrm{N}-\mathrm{W}-\mathrm{C}(1)$ & $103.2(3)$ \\
\hline $\mathrm{C}-\mathrm{W}-\mathrm{C}(1)$ & $74.1(3)$ \\
\hline $\mathrm{N}-\mathrm{W}-\mathrm{C}(2)$ & $94.8(2)$ \\
\hline $\mathrm{C}-\mathrm{W}-\mathrm{C}(2)$ & $108.9(3)$ \\
\hline $\mathrm{C}(1)-\mathrm{W}-\mathrm{C}(2)$ & $35.6(3)$ \\
\hline N-W-C(14) & $109.8(5)$ \\
\hline C-W-C(14) & $98.2(4)$ \\
\hline
\end{tabular}




\begin{tabular}{|c|c|}
\hline C(1)-W-C(14) & $146.3(6)$ \\
\hline C(2)-W-C(14) & $142.9(3)$ \\
\hline N-W-C(15) & $98.3(4)$ \\
\hline C-W-C(15) & $131.7(5)$ \\
\hline C(1)-W-C(15) & $146.0(6)$ \\
\hline C(2)-W-C(15) & $117.2(5)$ \\
\hline C(14)-W-C(15) & $34.3(5)$ \\
\hline N-W-C(11) & $122.7(5)$ \\
\hline C-W-C(11) & $142.9(4)$ \\
\hline $\mathrm{C}(1)-\mathrm{W}-\mathrm{C}(11)$ & $108.5(5)$ \\
\hline C(2)-W-C(11) & $85.2(4)$ \\
\hline C(14)-W-C(11) & $58.4(4)$ \\
\hline C(15)-W-C(11) & $37.7(5)$ \\
\hline N-W-C(13) & $142.2(4)$ \\
\hline C-W-C(13) & $88.7(4)$ \\
\hline C(1)-W-C(13) & $113.0(5)$ \\
\hline C(2)-W-C(13) & $120.9(4)$ \\
\hline C(14)-W-C(13) & $33.3(5)$ \\
\hline C(15)-W-C(13) & $56.2(5)$ \\
\hline C(11)-W-C(13) & $55.5(4)$ \\
\hline N-W-C(12) & $154.3(4)$ \\
\hline C-W-C(12) & $110.7(5)$ \\
\hline C(1)-W-C(12) & $96.0(4)$ \\
\hline C(2)-W-C(12) & $90.9(4)$ \\
\hline C(14)-W-C(12) & $55.3(5)$ \\
\hline C(15)-W-C(12) & $57.2(5)$ \\
\hline C(11)-W-C(12) & $32.9(4)$ \\
\hline C(13)-W-C(12) & $32.2(4)$ \\
\hline $\mathrm{O}(1)-\mathrm{N}-\mathrm{W}$ & $172.2(6)$ \\
\hline $\mathrm{O}(2)-\mathrm{C}-\mathrm{W}$ & $177.2(7)$ \\
\hline$C(12)-C(11)-C(15)$ & 104.1(10) \\
\hline$C(12)-C(11)-W$ & $74.6(5)$ \\
\hline$C(15)-C(11)-W$ & $70.1(5)$ \\
\hline $\mathrm{C}(13)-\mathrm{C}(12)-\mathrm{C}(11)$ & $112.0(14)$ \\
\hline$C(13)-C(12)-W$ & $73.9(6)$ \\
\hline $\mathrm{C}(11)-\mathrm{C}(12)-\mathrm{W}$ & $72.4(6)$ \\
\hline$C(12)-C(13)-C(14)$ & $109.9(13)$ \\
\hline$C(12)-C(13)-W$ & $73.9(6)$ \\
\hline$C(14)-C(13)-W$ & $71.0(6)$ \\
\hline
\end{tabular}




$\begin{array}{lc}\mathrm{C}(13)-\mathrm{C}(14)-\mathrm{C}(15) & 109.4(12) \\ \mathrm{C}(13)-\mathrm{C}(14)-\mathrm{W} & 75.7(5) \\ \mathrm{C}(15)-\mathrm{C}(14)-\mathrm{W} & 73.0(5) \\ \mathrm{C}(14)-\mathrm{C}(15)-\mathrm{C}(11) & 104.6(13) \\ \mathrm{C}(14)-\mathrm{C}(15)-\mathrm{W} & 72.8(5) \\ \mathrm{C}(11)-\mathrm{C}(15)-\mathrm{W} & 72.2(5) \\ \mathrm{C}(2)-\mathrm{C}(1)-\mathrm{W} & 72.8(4) \\ \mathrm{C}(1)-\mathrm{C}(2)-\mathrm{C}(21) & 124.5(7) \\ \mathrm{C}(1)-\mathrm{C}(2)-\mathrm{W} & 71.6(4) \\ \mathrm{C}(21)-\mathrm{C}(2)-\mathrm{W} & 117.1(4) \\ \mathrm{C}(22)-\mathrm{C}(21)-\mathrm{C}(2) & 126.2(7) \\ \mathrm{C}(22)-\mathrm{C}(21)-\mathrm{C}(25) & 111.1(7) \\ \mathrm{C}(2)-\mathrm{C}(21)-\mathrm{C}(25) & 122.5(7) \\ \mathrm{C}(21)-\mathrm{C}(22)-\mathrm{N}(2) & 124.3(8) \\ \mathrm{C}(21)-\mathrm{C}(22)-\mathrm{C}(23) & 111.8(7) \\ \mathrm{N}(2)-\mathrm{C}(22)-\mathrm{C}(23) & 123.8(7) \\ \mathrm{C}(24)-\mathrm{C}(23)-\mathrm{C}(22) & 104.8(7) \\ \mathrm{C}(23)-\mathrm{C}(24)-\mathrm{C}(25) & 107.6(8) \\ \mathrm{C}(21)-\mathrm{C}(25)-\mathrm{C}(24) & 104.0(7) \\ \mathrm{C}(31)-\mathrm{N}(2)-\mathrm{C}(41) & 119.3(10) \\ \mathrm{C}(31)-\mathrm{N}(2)-\mathrm{C}(22) & 116.3(12) \\ \mathrm{C}(41)-\mathrm{N}(2)-\mathrm{C}(22) & 118.3(7) \\ \mathrm{N}(2)-\mathrm{C}(31)-\mathrm{C}(32) & 111.6(16) \\ \mathrm{C}(42)-\mathrm{C}(41)-\mathrm{N}(2) & 113.6(4) \\ & \end{array}$

Symmetry transformations used to generate equivalent atoms: 
Table 4: Anisotropic displacement parameters $\left(\AA^{2} \times 10^{3}\right)$ for 11. The anisotropic displacement factor exponent takes the form: $-2 \pi^{2}\left[h^{2} a^{* 2} U^{11}+\ldots+2 h k a^{*} b^{*} U^{12}\right]$

\begin{tabular}{|c|c|c|c|c|c|c|}
\hline & $\mathrm{U}^{11}$ & $\mathrm{U}^{22}$ & $\mathrm{U}^{33}$ & $\mathrm{U}^{23}$ & $\mathrm{U}^{13}$ & $\mathrm{U}^{12}$ \\
\hline W & $51(1)$ & $53(1)$ & $44(1)$ & $-1(1)$ & $14(1)$ & $-4(1)$ \\
\hline $\mathrm{N}$ & $70(3)$ & $51(4)$ & $68(3)$ & $6(3)$ & $24(2)$ & $-2(3)$ \\
\hline $\mathrm{O}(1)$ & $128(5)$ & $51(4)$ & $135(6)$ & $-10(4)$ & $51(4)$ & $-6(3)$ \\
\hline $\mathrm{C}$ & $84(4)$ & $61(5)$ & $51(3)$ & $0(3)$ & $22(3)$ & $-15(3)$ \\
\hline $\mathrm{O}(2)$ & $154(6)$ & 103(6) & $70(3)$ & $15(3)$ & $40(4)$ & $-22(4)$ \\
\hline $\mathrm{C}(11)$ & 112(8) & $196(15)$ & $45(4)$ & $-18(6)$ & $18(4)$ & $-57(9)$ \\
\hline $\mathrm{C}(12)$ & $122(8)$ & $130(12)$ & $100(7)$ & $-65(8)$ & $11(6)$ & $-27(8)$ \\
\hline$C(13)$ & $94(7)$ & $139(12)$ & $91(6)$ & $-25(7)$ & $9(5)$ & $-52(8)$ \\
\hline$C(14)$ & $49(4)$ & $205(16)$ & $79(5)$ & $-17(8)$ & $7(3)$ & $-1(6)$ \\
\hline $\mathrm{C}(15)$ & $126(10)$ & $124(11)$ & 111(8) & $22(8)$ & $-62(8)$ & $-1(8)$ \\
\hline $\mathrm{C}(1)$ & $61(4)$ & $59(5)$ & $118(6)$ & $21(4)$ & $24(4)$ & $9(3)$ \\
\hline$C(2)$ & $51(3)$ & $61(5)$ & $82(4)$ & $-5(3)$ & $23(3)$ & $0(3)$ \\
\hline $\mathrm{C}(21)$ & $45(3)$ & $60(4)$ & $74(4)$ & $0(3)$ & $17(3)$ & $0(3)$ \\
\hline$C(22)$ & $62(3)$ & $60(5)$ & $95(5)$ & $2(4)$ & $33(3)$ & $3(3)$ \\
\hline$C(23)$ & $77(5)$ & $95(7)$ & $116(7)$ & $19(6)$ & $24(5)$ & $-20(5)$ \\
\hline $\mathrm{C}(24)$ & $62(4)$ & $81(7)$ & $142(9)$ & $-11(6)$ & $13(5)$ & $-18(4)$ \\
\hline$C(25)$ & $66(4)$ & $99(7)$ & $90(5)$ & $0(5)$ & $4(3)$ & $-25(5)$ \\
\hline $\mathrm{N}(2)$ & 152(8) & $80(6)$ & $89(5)$ & $-8(4)$ & $57(5)$ & $-4(5)$ \\
\hline
\end{tabular}


